\title{
LOCALIZATION OF PHENCYCLIDINE BINDING SITES ON $\alpha$ AND $\beta$ SUBUNITS OF THE NICOTINIC ACETYLCHOLINE RECEPTOR FROM TORPEDO OCELLATA ELECTRIC ORGAN USING AZIDO PHENCYCLIDINE ${ }^{1}$
}

\author{
R. HARING, Y. KLOOG, AND M. SOKOLOVSKY ${ }^{2}$ \\ Department of Biochemistry, The George S. Wise Faculty of Life Sciences, Tel Aviv University, Tel Aviv 69978, Israel
}

Received January 27, 1983; Revised September 13, 1983; Accepted September 13, 1983

\begin{abstract}
A photolabile derivative of phencycliding (PCP), azido phencyclidine (AZ-PCP), was synthesized and used to localize PCP binding sites on the acetylcholine receptor from Torpedo ocellata electric organ. In the dark, the binding of micromolar concentrations of $\left[{ }^{3} \mathrm{H}\right] \mathrm{AZ}-\mathrm{PCP}$ to a receptor-enriched membrane preparation fits a single dissociation constant $\left(K_{d}=2.65 \mu \mathrm{M}\right)$ and is very similar to the binding of $\left[{ }^{3} \mathrm{H}\right] \mathrm{PCP}$. The agonist carbamylcholine increases the association rate (and the affinity) of these ligands to the receptor, but it does not alter the total number of available binding sites.

Following UV irradiation and gel electrophoresis, $\left[{ }^{3} \mathrm{H}\right] \mathrm{AZ}-\mathrm{PCP}$ was found to label specifically the $\alpha$ and $\beta$ subunits of the receptor. The labeling of the $\alpha$ subunit band was heavier, and it was inhibited by tetracaine and PCP but not by $\alpha$-bungarotoxin $(\alpha$-Bgt). The addition of carbamylcholine enhanced the labeling of the $\beta$ subunit; this effect was diminished by $\alpha$-Bgt. The labeling of the $\beta$ subunit was also inhibited by tetracaine and PCP. The effect of carbamylcholine, which binds to the $\alpha$ subunit, could be the result of an induced conformational change, which is propagated to the $\beta$ subunit and increases its labeling by $\left[{ }^{3} \mathrm{H}\right] \mathrm{AZ}-\mathrm{PCP}$.

A simple model which accommodates the binding and photoaffinity labeling data is described. According to the model, the high affinity PCP binding site is located between the $\alpha$ and $\beta$ receptor subunits, and the drug thus becomes attached simultaneously to both. Hypothetical overlapping recognition sites for PCP on these receptor subunits would allow binding (and labeling) with increased affinity in the presence of carbamylcholine with no increase in the number of available sites.
\end{abstract}

Phencyclidine (PCP) is a synthetic arylcyclohexylamine with multiple actions on animals and humans ( $\mathrm{Pe}-$ tersen and Stillman, 1978). Its activity as a general anesthetic (Johnston et al., 1959) and its effects on animal behavior (Petersen and Stillman, 1978) have been known for many years. Interest in PCP has increased in recent years because of frequent abuse of the drug, with harmful consequences. With the development of sensitive binding assays for receptors, studies have indicated that PCP interferes with the binding of several drugs to their muscarinic (Kloog et al., 1977; Gabrielevitz et al., 1980; Vincent et al., 1978; Aronstam et al., 1980), opiate (Vincent et al., 1978), and other receptors. The existence

\footnotetext{
${ }^{1}$ We wish to thank Professor A. Kalir for the synthesis of the unlabeled azido derivative and for his invaluable advice, and Mrs. R. Galron for her excellent technical assistance. This work was supported in part by a grant from the Israel Commission for Basic Research.

${ }^{2}$ To whom correspondence should be addressed.
}

of specific high affinity binding sites of PCP in rat brain tissue has also been demonstrated (Vincent et al., 1979; Zukin and Zukin, 1979).

It is not known whether the anticholinergic activity of PCP (Maayani et al., 1974; Paster et al., 1974; Kloog et al., 1977; Kalir et al., 1978) contributes to its psychotic activity. The drug is a potent inhibitor of butyrylcholinesterase (Maayani et al., 1974; Kloog et al., 1977) and can block both muscarinic (Maayani et al., 1974; Kloog et al., 1977) and nicotinic (Kloog et al., 1977; Albuquerque et al., 1980) responses in tissues. It is now recognized that PCP can act as a noncompetitive blocker of the nicotinic response to acetylcholine (AcCho). This finding was based on electrophysiological (Albuquerque et al., 1980) as well as on binding (Albuquerque et al., 1980; Eldefrawi et al., 1980; Kloog et al., 1979, 1980) data indicating that PCP blocks the cholinergic ionophore. PCP does not inhibit binding of the specific nicotinic neurotoxin $\alpha$-bungarotoxin ( $\alpha$-Bgt) (Kloog et al., 1980), 
and binds to specific binding sites in Torpedo electric organ membrane that are distinct from AcCho binding sites (Eldefrawi et al., 1980; Kloog et al., 1979, 1980; Oswald and Changeux, 1981a). As with other noncompetitive blockers of AcCho (Oswald and Changeux, 1981a; Kloog et al., 1979; Elliott and Raftery, 1979), the binding of PCP is enhanced by the agonist carbamylcholine (Eldefrawi et al., 1980; Oswald and Changeux, 1981a). Thus, all of the data suggest that PCP interacts directly with the acetylcholine receptor (AcChoR).

The AcChoR from Torpedo electric organ is a pentamer composed of four different polypeptides with apparent molecular weights of $\sim 40,000(\alpha), \sim 50,000(\beta)$, $\sim 60,000(\gamma)$, and $\sim 65,000(\delta)$ (Karlin, 1980; Conti-Tronconi and Raftery, 1982). Two $\alpha$ subunits are present in each pentamer and on them are located the binding sites for AcCho and snake toxins (Karlin, 1980; Conti-Tronconi and Raftery, 1982). If PCP does indeed act directly on the AcChoR, it could be expected to interact with at least one of the receptor subunits. In order to localize the site on the AcChoR to which PCP would bind, we have synthesized a photolabile derivative of PCP, azido phencyclidine (AZ-PCP), and used it to label receptors on membranes purified from electric organ of Torpedo ocellata. Azido derivatives of drugs have the advantage that they can be used under two sets of conditions: those in which they bind reversibly and those in which they irreversibly inactivate their binding sites.

We report here on the specific labeling of $\alpha$ and $\beta$ subunits of the AcChoR from T. ocellata by $\left[{ }^{3} \mathrm{H}\right] \mathrm{AZ}-\mathrm{PCP}$. The simplest explanation accommodating both the binding and the labeling data obtained is that the binding site for PCP is located close to the physical connection between the $\alpha$ and $\beta$ subunits, enabling the ligand to attach simultaneously to both.

\section{Materials and Methods}

Phencyclidine and 3-aminophencyclidine (1-(1-(3aminophenyl)-cyclohexyl)piperidine) were prepared as described (Kalir et al., 1978). 3-Azido phencyclidine (1(1-(3-azidophenyl)-cyclohexyl)piperidine) was prepared from 3-aminophencyclidine by dissolving it in $1 \mathrm{M}$ hydrochloric acid, treating it with $\mathrm{KNO}_{2}$, and then adding $\mathrm{NaN}_{3}$ as described previously (Amitai et al., 1982). Compounds were characterized by $\mathrm{C}, \mathrm{H}, \mathrm{N}$ analysis and infrared spectroscopy and were tested for purity $(>98 \%)$ by two thin layer chromatography systems (Kalir et al., 1978). The melting point of the AZ-PCP (free base) was 51 to $52^{\circ} \mathrm{C}$.

$\left[{ }^{3} \mathrm{H}\right] \mathrm{PCP}(22.5 \mathrm{Ci} / \mathrm{mmol})$ was prepared by catalytic tritium exchange and was tested for purity $(>98 \%)$ as described elsewhere (Kalir et al., 1978), using a synthetic technique analogous to that described by Kalir et al. (1975). [ $\left.{ }^{3} \mathrm{H}\right] \mathrm{AZ}-\mathrm{PCP}(27.1 \mathrm{Ci} / \mathrm{mmol})$ was prepared by diazotation of tritiated aminophencyclidine followed by reaction with sodium azide. Purity was $>99 \%$.

Decamethonium, hexamethonium, tetracaine, carbamylcholine, $d$-tubocurarine, $\alpha$-Bgt, and quinacrine were obtained from Sigma.

Tissue preparation. Membranes rich in acetylcholine receptor were prepared from $150 \mathrm{gm}$ of freshly dissected electric organ of live T. ocellata (caught off the Mediter- ranean coast of Tel-Aviv), as described by Saitoh et al. (1980). The electric organs were homogenized in $50 \mathrm{mM}$

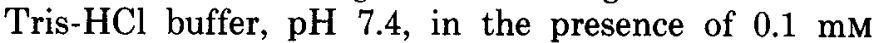
phenylmethylsulfonyl fluoride, $3 \mathrm{mM}$ EDTA, $1 \mathrm{mM}$ EGTA, 5 units $/ \mathrm{ml}$ of aprotinine and $5 \mu \mathrm{g} / \mathrm{ml}$ of pepstatin A. The pellet $\left(\mathrm{p}_{3}\right)$ was resuspended in $36 \%$ sucrose $(\mathrm{w} /$ w), sonicated, and then layered on top of $41 \%$ sucrose and centrifuged at $43,000 \mathrm{rpm}$ in a Beckman $45 \mathrm{Ti}$ rotor for $3 \mathrm{hr}$. The membrane fragments located at the interface were collected and further purified by alkaline treatment (Neubig et al., 1979). The $\mathrm{pH}$ was adjusted to 11 using $1 \mathrm{M} \mathrm{NaOH}$, and the mixture was incubated for 60 min at room temperature and then centrifuged at $4^{\circ} \mathrm{C}$ for $30 \mathrm{~min}$ at $194,000 \times \mathrm{g}$. The pellet was resuspended in the homogenization buffer. The membranes were either used immediately or stored at $-70^{\circ} \mathrm{C}$ until use. Specific $\alpha$-Bgt binding sites were determined by means of the DEAE filter disk assay (Schmidt and Raftery, 1973), using ${ }^{125} \mathrm{I}$-labeled $\alpha$-Bgt. The purified AcChoR-rich membrane fragments had a specific activity of 1.6 to $2.0 \mathrm{nmol} /$ $\mathrm{mg}$.

Binding assays. Binding of $\left[{ }^{3} \mathrm{H}\right] \mathrm{PCP}$ and $\left[{ }^{3} \mathrm{H}\right] \mathrm{AZ}-\mathrm{PCP}$ was assayed by both the centrifugation and the filtration methods. The latter ligand was assayed in the dark. In the centrifugation method, aliquots $(50 \mu \mathrm{l})$ of membrane preparation containing 40 to $60 \mu \mathrm{g}$ of protein were pipetted into plastic microfuge tubes containing $100 \mu \mathrm{l}$ of buffered $\left[{ }^{3} \mathrm{H}\right] \mathrm{PCP}$ or $\left[{ }^{3} \mathrm{H}\right] \mathrm{AZ}-\mathrm{PCP}$ and either $50 \mu \mathrm{l}$ of Torpedo physiological buffer $(250 \mathrm{mM} \mathrm{NaCl}, 5 \mathrm{mM} \mathrm{KCl}$, $2 \mathrm{mM} \mathrm{MgCl}_{2}, 2 \mathrm{mM} \mathrm{CaCl}, 50 \mathrm{mM}$ sodium phosphate, $\mathrm{pH}$ 7.0) or $50 \mu \mathrm{l}$ of buffer containing ligand. Following incubation at $25^{\circ} \mathrm{C}$ for $30 \mathrm{~min}$ to reach equilibrium, the tubes were centrifuged for $4 \mathrm{~min}$ (Eppendorf microfuge, model 5415). The supernatant was sampled for determination of free ligand and was discarded. The pellets were washed rapidly three times with $1 \mathrm{ml}$ of ice-cold buffer. The tip of the tube was cut and its contents collected in scintillation vials containing $4 \mathrm{ml}$ of scintillation liquid (Hydro-Luma, Lumac Systems), stirred with a vortex, and kept at room temperature for $24 \mathrm{hr}$. Counting was carried out in a Packard Prias-PL, with a counting efficiency of $40 \%$.

When binding was assayed by the filtration method the reaction was terminated by filtration on Whatman $\mathrm{GF} / \mathrm{C}$ glass filters which were washed twice with $3 \mathrm{ml}$ of buffer. Adsorbance of the labeled drugs to the filters in the absence of membranes was low ( $10 \%$ of total binding). It was measured both in the absence and in the presence of $5 \times 10^{-4} \mathrm{M}$ unlabeled PCP and was subtracted from the counts of corresponding samples measured in the presence of membranes.

All assays were carried out in triplicate, together with triplicate control samples containing $5 \times 10^{-4} \mathrm{M}$ unlabeled PCP. Specific binding was calculated as the total minus the nonspecific (the latter is the binding that occurs in the presence of $5 \times 10^{-4} \mathrm{M}$ unlabeled PCP).

Competition experiments using $1 \mu \mathrm{M}\left[{ }^{3} \mathrm{H}\right] \mathrm{PCP}$ or $\left[{ }^{3} \mathrm{H}\right]$ AZ-PCP and the various drugs were carried out by the centrifugation method as described above. The dissociation constants $\left(K_{d}\right)$ and the maximal binding capacity $\left(B_{\max }\right)$ of the unlabeled derivatives were obtained by Scatchard plots by a linear least square regression analy- 
sis. Protein was determined by the Lowry method using bovine serum albumin as a standard.

Photoaffinity labeling. AcChoR-enriched membranes were incubated with 0.5 to $1.0 \mu \mathrm{M}\left[{ }^{3} \mathrm{H}\right] \mathrm{AZ}-\mathrm{PCP}$ in the dark for $30 \mathrm{~min}$ under conditions similar to those described for the binding assays, except that the volume of the reaction mixture was $300 \mu \mathrm{l}$. The incubation was carried out in the presence and absence of various agents. For nonspecific binding $1 \times 10^{-3} \mathrm{M}$ PCP or tetracaine was added. Following incubation, samples were withdrawn for determination of total binding. The reaction mixture was then photolyzed with a long wave ultraviolet spotlight lamp (Thomas Scientific Apparatus, model B$100 \mathrm{~A})$ at a distance of $5 \mathrm{~cm}\left(1500 \mu \mathrm{W} / \mathrm{cm}^{2}\right)$ with continuous stirring for $4 \mathrm{~min}$. After photolysis, membranes were either precipitated and washed four times with Tris$\mathrm{HCl}$ buffer $(50 \mathrm{~mm}, \mathrm{pH} 7.4)$ or diluted 500 -fold in the Tris buffer and filtered on GF/C filters after $60 \mathrm{~min}$ of incubation at room temperature. The amount of specifically attached labeled AZ-PCP after precipitation and washing or after dilution was taken as a reflection of the extent of covalent binding of the ligand. Precipitated washed membranes were used for gel electrophoresis. In control experiments membranes were photolyzed under the standard conditions in the absence of drugs and then incubated with $\left[{ }^{3} \mathrm{H}\right] \mathrm{PCP}$. No change in binding of the labeled drug was observed. Moreover, photolysis of $\left[{ }^{3} \mathrm{H}\right]$ PCP instead of $\left[{ }^{3} \mathrm{H}\right] \mathrm{AZ}-\mathrm{PCP}$ under these conditions did not result in any irreversible binding $(<1 \%$ of the reversible bound $\mathrm{PCP}$ at equilibrium).

We also examined the effect of varying some of the photolysis conditions in the case of $\left[{ }^{3} \mathrm{H}\right] \mathrm{AZ}-\mathrm{PCP}$ membrane complexes, such as the time of photolysis and the distance of the membranes from the photolysis source. The highest specific irreversible binding of $\left[{ }^{3} \mathrm{H}\right] \mathrm{AZ}-\mathrm{PCP}$ was observed under the conditions described above.

Gel electrophoresis. The SDS polyacrylamide gel electrophoresis procedure was carried out as described by Laemmli (1970). Membrane samples were dissolved 1:1 $(\mathrm{v} / \mathrm{v})$ in $2 \times$ sample buffer $(52.5 \mathrm{~mm}$ Tris- $\mathrm{HCl}, \mathrm{pH} 6.8$, $2 \%$ SDS, $10 \%$ glycerol, $5 \% 2$-mercaptoethanol) and applied to polyacrylamide slab gels $(8.0 \%)$. Proteins were electrophoresed at 15 to $20 \mathrm{~mA} / \mathrm{slab}$ for $3 \mathrm{hr}$, then stained with $0.2 \%$ Coomassie brilliant blue in $50 \%$ methanol and $7 \%$ acetic acid and destained in $7.5 \%$ acetic acid. Gels were sliced into 1-mm sections with a gel slicer. Each slice was digested in $5 \mathrm{ml}$ of $6 \%$ Protosol (New England Nuclear) in Hydro Luma in a closed scintillation vial. Radioactivity was determined after $24 \mathrm{hr}$ by liquid scintillation spectrometry. Other gels were prepared for fluorography as described (Bonner and Laskey, 1974), and exposed to an Agfa $x$-ray film (Curix RP-2) which was developed 30 days later (Kodak $\mathrm{x}$-ray developer and fixer).

\section{Results}

Common binding sites for $\left[{ }^{3} \mathrm{H}\right] P C P$ and $\left[{ }^{3} \mathrm{H}\right] A Z-P C P$. Previous studies have shown that $\left[{ }^{3} \mathrm{H}\right] \mathrm{PCP}$ (Kloog et al., 1979, 1980; Oswald and Changeux, 1981a) and its amino derivative (Kloog et al., 1980) bind to AcChoR-enriched membranes at sites distinct from the AcCho binding site. In order to characterize and localize these binding sites further, a photolabile derivative of phencyclidine, azido phencyclidine, was synthesized. The specificity of the new derivative was examined in binding experiments performed in the dark, i.e., under conditions in which the AZ-PCP is stable and binds reversibly to its sites. Its binding characteristics were compared to those of PCP binding to the AcChoR-enriched membranes.

Binding of $\left[{ }^{3} \mathrm{H}\right] \mathrm{PCP}$ and $\left[{ }^{3} \mathrm{H}\right] \mathrm{AZ}-\mathrm{PCP}$ to AcChoRenriched membranes was assayed by the centrifugation method (Kloog et al., 1979). Significant binding of both was observed at the micromolar concentration range. The total binding of $\left[{ }^{3} \mathrm{H}\right] \mathrm{AZ}-\mathrm{PCP}$ was about 3 to 4 times higher than that of $\left[{ }^{3} \mathrm{H}\right] \mathrm{PCP}$. The higher binding capacity of $\left[{ }^{3} \mathrm{H}\right] \mathrm{AZ}-\mathrm{PCP}$ can be explained by the fact that its nonspecific binding is higher than that of $\left[{ }^{3} \mathrm{H}\right] \mathrm{PCP}$; in the presence of $5 \times 10^{-4} \mathrm{M}$ unlabeled PCP (nonspecific binding), binding of $\left[{ }^{3} \mathrm{H}\right] \mathrm{AZ}-\mathrm{PCP}(0.1$ to $15 \mu \mathrm{M})$ was about 3 to 4 times higher than that of $\left[{ }^{3} \mathrm{H}\right] \mathrm{PCP}$. The nonspecific binding of either ligand was linearly dependent on its concentration and did not change when measured in the presence of other ligands known to interact with PCP binding sites (not shown).

As shown in Figure 1, the specific binding of $\left[{ }^{3} \mathrm{H}\right] \mathrm{PCP}$
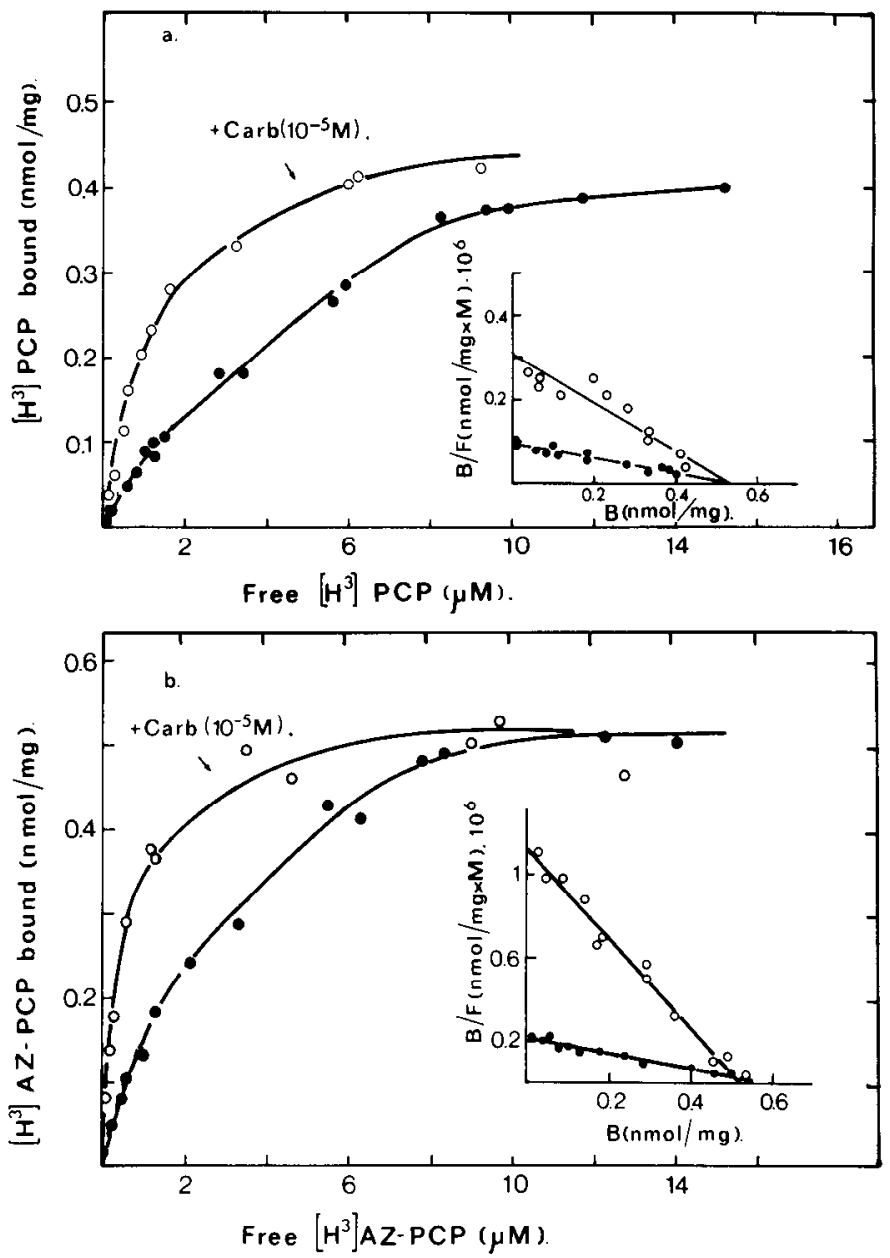

Figure 1. Specific binding (total minus nonspecific binding) of $\left[{ }^{3} \mathrm{H}\right] \mathrm{PCP}(a)$ and $\left[{ }^{3} \mathrm{H}\right] \mathrm{AZ}-\mathrm{PCP}(b)$ as a function of ligand concentration. Binding was determined in the absence $(\mathrm{O}-\mathrm{O})$ and in the presence (-) of $10^{-5} \mathrm{M}$ carbamylcholine as described under "Materials and Methods." Insets, Scatchard plot of the same data. 
and $\left[{ }^{3} \mathrm{H}\right] \mathrm{AZ}-\mathrm{PCP}$ (defined as the total minus nonspecific binding) was similar. Binding of the labeled drugs to the membranes was saturable in each case. Replotting the binding data according to Scatchard yielded linear curves (Fig. 1, insets) from which the dissociation constants and the binding capacities of the ligands were calculated. The binding capacity of the AcChoR-enriched membranes was found to be similar for the two ligands (Table I), but the dissociation constant of $\left[{ }^{3} \mathrm{H}\right] \mathrm{AZ}-\mathrm{PCP}(2.6 \mu \mathrm{M})$ was about 3 times lower than that of $\left[{ }^{3} \mathrm{H}\right] \mathrm{PCP}$. It should be noted that the experiments with $\left[{ }^{3} \mathrm{H}\right] \mathrm{AZ}-\mathrm{PCP}$ were performed at lower concentrations than those with $\left[{ }^{3} \mathrm{H}\right] \mathrm{PCP}$ (Fig. 1) because of the higher affinity of the former drug. The actual nonspecific binding of $\left[{ }^{3} \mathrm{H}\right] \mathrm{AZ}-\mathrm{PCP}$ is, therefore, similar to that of $\left.l^{3} \mathrm{H}\right] \mathrm{PCP}$.

Several binding characteristics typical of PCP have been described, among them its enhancement in the presence of cholinergic agonists (Eldefrawi et al., 1980; Oswald and Changeux, 1981a) and its competitive displacement by aminated local anesthetics and other noncompetitive blockers of AcCho (Eldefrawi et al., 1980; Kloog et al., 1980; Oswald and Changeux, 1981a). These binding characteristics were found to be preserved in the azido derivative; carbamylcholine at $10^{-5} \mathrm{M}$ strongly enhanced the binding of $\left[{ }^{3} \mathrm{H}\right] \mathrm{AZ}-\mathrm{PCP}$ as well as that of $\left[{ }^{3} \mathrm{H}\right] \mathrm{PCP}$ (Fig. 1, $a$ and $b$ ). Quinacrine and the local anesthetic tetracaine inhibited their binding (Fig. 2). In addition, PCP inhibited $\left[{ }^{3} \mathrm{H}\right] \mathrm{AZ}-\mathrm{PCP}$ binding to the receptor. It should be noted that, although carbamylcholine increased the affinity of the labeled ligands toward their binding sites, it did not increase their binding capacity (Fig. 1, Table I). The increased affinity is at least partly due to the increased rate of association of $\left[{ }^{3} \mathrm{H}\right] \mathrm{PCP}$ to the receptor-enriched membrane, which in turn is dependent on the concentration of the agonist (Eldefrawi et al., 1980).

The dose-dependent enhancement of $\left[{ }^{3} \mathrm{H}\right] \mathrm{PCP}$ binding induced by carbamylcholine is shown in Figure 3. The effect is observed already at $0.1 \mu \mathrm{M}$ carbamylcholine, reaches maximal level at $\sim 1 \mu \mathrm{M}$, and does not fade even at $10^{-3} \mathrm{M}$. When $1 \mu \mathrm{M} \alpha$-Bgt is present in the incubation medium, the carbamylcholine-induced enhancement of $\left[{ }^{3} \mathrm{H}\right] \mathrm{PCP}$ binding is blocked, but there is no effect on the basal level of PCP binding (Fig. $3 a$ ). Moreover, the presence of the toxin did not increase the inhibition of $\left[{ }^{3} \mathrm{H}\right] \mathrm{PCP}$ binding by PCP. These data confirm previous findings suggesting that PCP does not bind to the $\alpha$-Bgt

TABLE I

Effect of carbamylcholine on the binding characteristics of PCP and $A Z-P C P$

The average values of binding parameters determined in three to five separate experiments each performed in triplicate are given. Values for $K_{d}$ and maximal hinding capacity ( $B_{\max }$ ) were calculated as described under "Materials and Methods."

\begin{tabular}{lccccc}
\hline & \multicolumn{2}{c}{ Carbamylcholine Absent } & & \multicolumn{2}{c}{$\begin{array}{c}\text { Carbamylcholine }\left(10^{-5} \mathrm{M}\right) \\
\text { Present }\end{array}$} \\
\cline { 2 - 3 } \cline { 5 - 6 } & $K_{d}$ & $B_{\max }$ & & $K_{d}$ & $B_{\max }$ \\
\hline$\mu \mathrm{M}$ & $p m o l / m g$ & & $\mu M$ & $p m o l / m g$ \\
PCP & $6.9 \pm 3.1$ & $606 \pm 144$ & & $1.7 \pm 0.8$ & $555 \pm 126$ \\
AZ-PCP & $2.65 \pm 0.7$ & $506 \pm 178$ & & $0.4 \pm 0.07$ & $446 \pm 33$ \\
\hline
\end{tabular}

binding sites (AcCho binding sites) (Kloog et al., 1980). Since both carbamylcholine and $\alpha$-Bgt bind to the AcCho binding site on the $\alpha$ subunit of the AcChoR (Karlin, 1980 ), the data indicate that the enhancement of $\left[{ }^{3} \mathrm{H}\right]$ PCP binding to its sites is mediated via the $\alpha$ subunit. Like carbamylcholine, the agonist decamethonium enhanced $\left[{ }^{3} \mathrm{H}\right] \mathrm{PCP}$ binding at the micromolar range (Fig. $3 b$ ). However, the higher concentrations of this drug inhibited $\left.{ }^{3} \mathrm{H}\right] \mathrm{PCP}$ binding. The enhancement effect, but not the inhibition phase, of decamethonium was blocked by $1 \mu \mathrm{M} \alpha$-Bgt (Fig. $3 b$ ). Furthermore, $\alpha$-Bgt did not enhance the inhibition of $\left[{ }^{3} \mathrm{H}\right] \mathrm{PCP}$ binding by decamethonium. Similar effects on $\left[{ }^{3} \mathrm{H}\right] \mathrm{PCP}$ binding were $\mathrm{ob}$ served with the antagonists hexamethonium (Fig. $3 b$ ) and $d$-tubocurarine (Fig. $3 c$ ).

The data on the reversible binding of $\left[{ }^{3} \mathrm{H}\right] \mathrm{PCP}$ and $\left[{ }^{3} \mathrm{H}\right] \mathrm{AZ}-\mathrm{PCP}$ - the competitive nature of their binding, the similarity in the number of binding sites, the inhibition of their binding by local anesthetics, and the enhancement of their binding induced by cholinergic drugs-all strongly suggest that the two ligands bind to the same binding sites on the AcChoR. It follows that AZ-PCP can be used as a photoaffinity probe for PCP binding sites.

Photoaffinity labeling of the AcChoR with AZ-PCP. The dark-stable property of AZ-PCP enables the characterization of its reversible binding properties. It also allows experimental separation of the binding reaction from the photoinactivation process. Conditions for the latter were selected empirically and are described under "Materials and Methods." The amount of labeled drug which remained attached to the membranes after photolysis was compared with the amount of reversible bound $\left[{ }^{3} \mathrm{H}\right] \mathrm{AZ}-\mathrm{PCP}$.

Figure 4 shows that 20 to $30 \%$ of the specifically bound drug remained attached to the membrane after UV irradiation and dilution. At higher concentrations of $\left[{ }^{3} \mathrm{H}\right]$ AZ-PCP, more of the drug remained attached. Similar results were obtained when the membranes were precipitated and washed (Fig. 4). More than $95 \%$ of the specifically bound $\left[{ }^{3} \mathrm{H}\right] \mathrm{AZ}-\mathrm{PCP}$ disappeared when the membranes were not irradiated and diluted, indicating that the irreversible attachment of the drug to the membranes was indeed a result of the photolysis. Attempts to increase the yields of $\left[{ }^{3} \mathrm{H}\right] \mathrm{AZ}-\mathrm{PCP}$ photoinactivated binding sites by varying the time of exposure to UV light and by using a short wave UV lamp (245 $\mathrm{nm}$ ) failed. Nevertheless, the high specific activity of the azido derivative and enrichment of the membranes in specific binding sites enabled us to further characterize those binding sites to which the drug had been covalently attached.

Identification of AZ-PCP labeled receptor subunits. To identify the polypeptides in the AcChoR-enriched membranes to which $\left[{ }^{3} \mathrm{H}\right] \mathrm{AZ}-\mathrm{PCP}$ is attached, SDS polyacrylamide gel electrophoresis was used. Membranes were subjected to electrophoresis after photoaffinity labeling under the standard conditions. The gels were stained, destained, and either cut for counting or used for fluorography.

A typical fluorogram is shown in Figure 5. Several bands are observed, corresponding to polypeptides with 

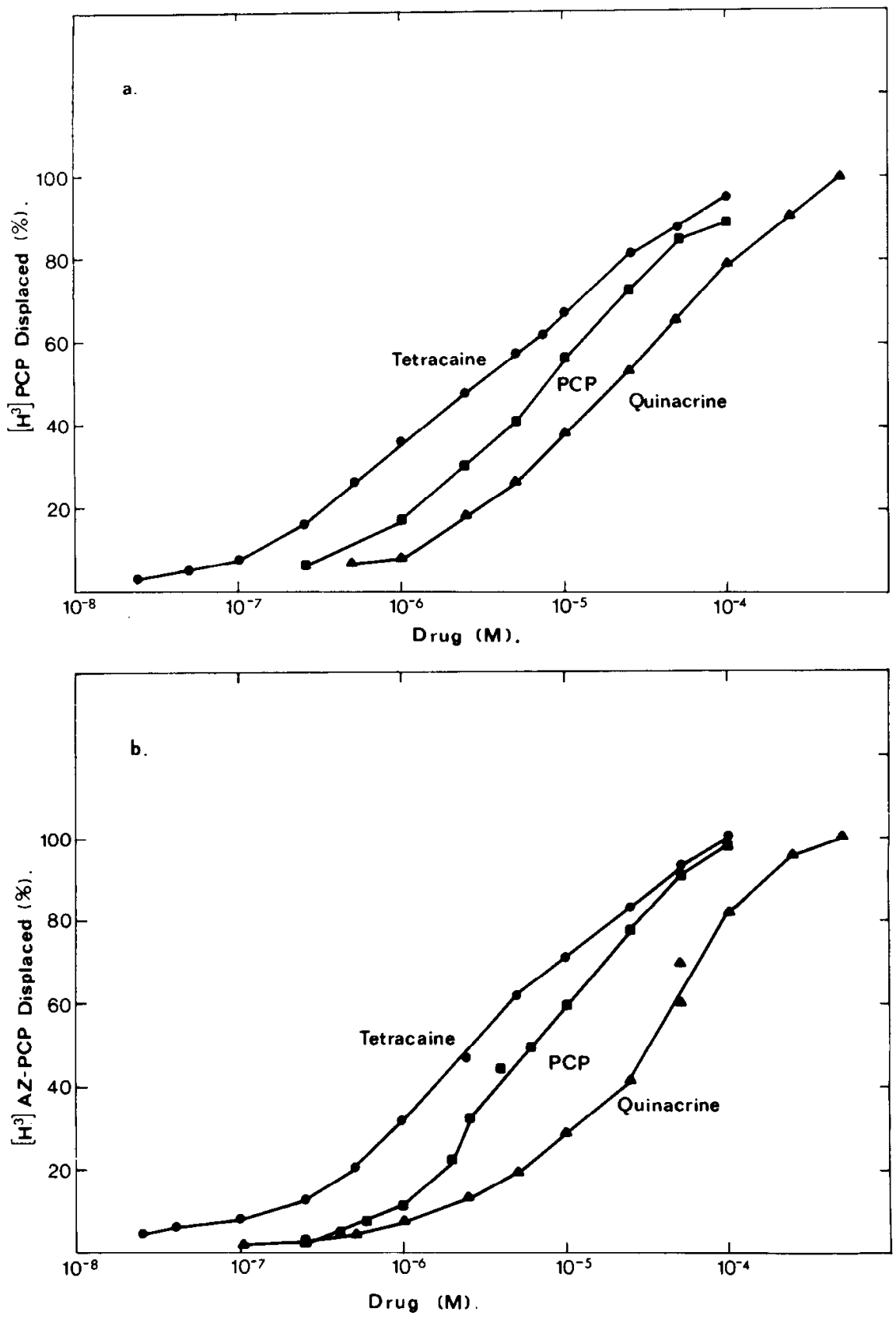

Figure 2. Inhibition of binding of $\left[{ }^{3} \mathrm{H}\right] \mathrm{PCP}(a)$ and $\left[{ }^{3} \mathrm{H}\right] \mathrm{AZ}-\mathrm{PCP}(b)$. Aliquots of membrane preparation were incubated with $1 \mu \mathrm{M}{ }^{3} \mathrm{H}$-ligand and with the unlabeled drugs at the concentrations indicated. Binding was determined (five separate determinations, each in triplicate) by the centrifugation assay as described under "Materials and Methods." Data are presented as percentage inhibition of the specific binding of ${ }^{3} \mathrm{H}$-ligand by PCP $(\square)$, tetracaine (O), and quinacrine $(\Delta)$.

apparent molecular weight of $92,000,80,000,65,000$, $55,000,50,000$, and 40,000 . The last four correspond to the well described AcChoR subunits (Karlin, 1980; Conti-Tronconi and Raftery, 1982). In the presence of $10^{-3} \mathrm{M}$ PCP (Fig. $5 \mathrm{~A}$ ) or $10^{-3} \mathrm{M}$ tetracaine (Fig. $5 \mathrm{~B}$ ), the labeling intensity of all of the polypeptides observed in the gel was strongly reduced.

The 40,000-dalton subunit was the most intense band observed when the membranes were labeled in the absence of carbamylcholine (Fig. $5, A$ and $B$ ). On the other hand, in the presence of $10^{-5} \mathrm{M}$ (Fig. $5 A$ ) or $10^{-4} \mathrm{M}$ (Fig. $5 B$ ) carbamylcholine, the intensity of the 50,000 -dalton subunit was enhanced, while no apparent effect on the other polypeptides was observed. Similar enhancement was induced by $d$-tubocurarine $\left(10^{-6} \mathrm{M}\right)(\mathrm{Fig} .5 B)$. The data presented in Figure 6 demonstrate that the enhancement by carbamylcholine of the labeling of the 50,000 dalton subunit can be blocked by $1 \mu \mathrm{M} \alpha$-Bgt. Like the fluorograms (Fig. 5), direct counting of the gels (Fig. 6) showed that the 40,000 -dalton subunit is labeled both in 

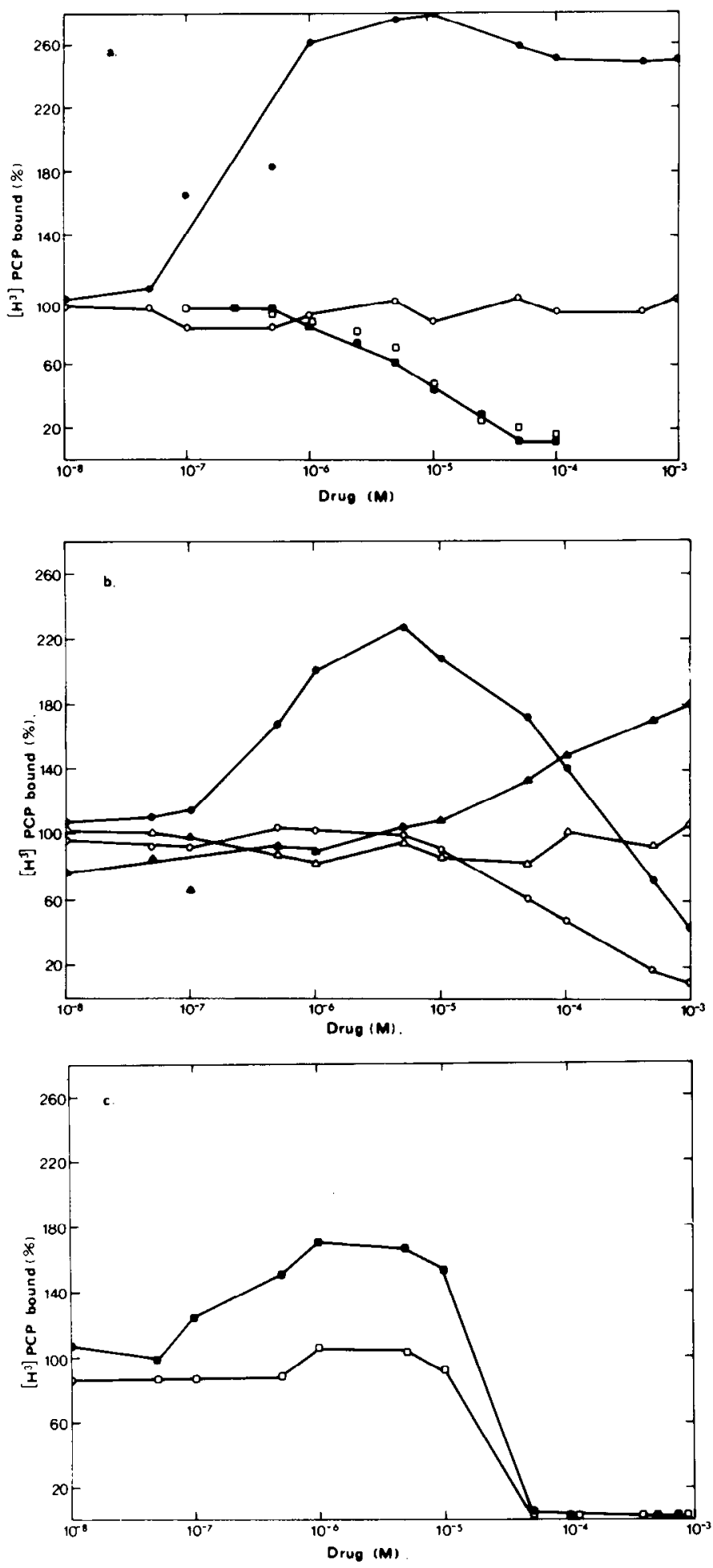

Figure 3. Inhibition and enhancement of $\left[{ }^{3} \mathrm{H}\right] \mathrm{PCP}$ binding by various agents. Membranes were incubated in the presence of $1 \mu \mathrm{M}\left[{ }^{3} \mathrm{H}\right] \mathrm{PCP}$ and the unlabeled drug at various concentrations as indicated, and binding was determined as described for Figure 4. Data are expressed as a percentage of the specific binding of $1 \mu \mathrm{M}\left[{ }^{3} \mathrm{H}\right] \mathrm{PCP}, a$, Binding in the presence of carbamylcholine (O), carbamylcholine $+1 \mu \mathrm{M} \alpha$-Bgt (O), PCP (a), and PCP $+1 \mu \mathrm{M} \alpha$-Bgt ( $\square$ ). $b$, Binding in the presence of decamethonium (O), decamethonium $+1 \mu \mathrm{M} \alpha$-Bgt $(O)$, hexamethonium ( $\mathbf{A}$ ), and hexamethonium $+1 \mu \mathrm{M} \alpha$-Bgt $(\Delta)$. $c$, Binding in the presence of $d$-tubocurarine $(\square)$ and $d$-tubocu-

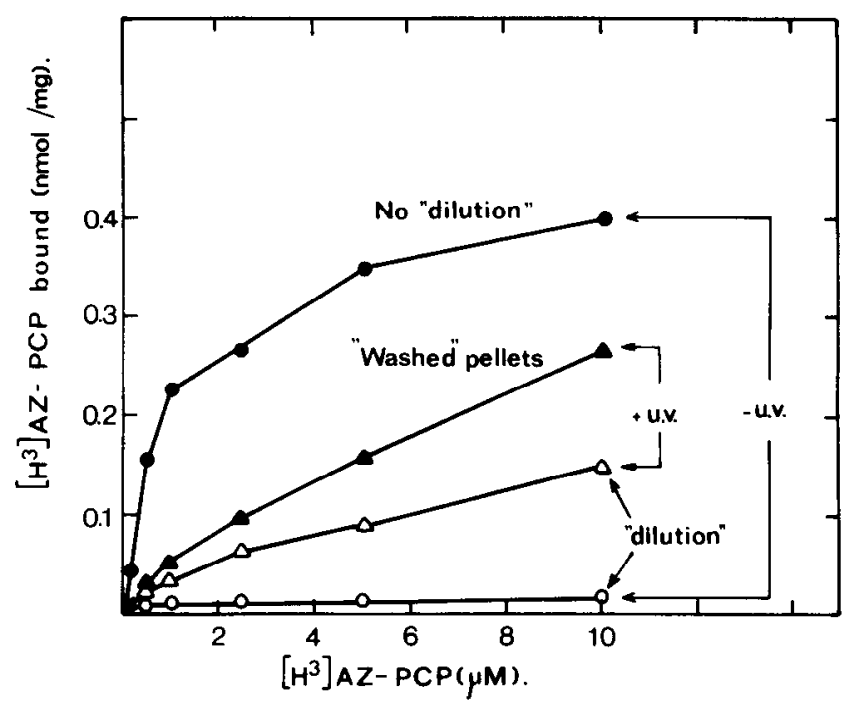

Figure 4. Irreversible binding of $\left[{ }^{3} \mathrm{H}\right] \mathrm{AZ}-\mathrm{PCP}$ to receptorenriched membranes. Membranes $(100 \mu \mathrm{g})$ were incubated for $30 \mathrm{~min}$ in the dark with the labeled ligand in the absence or in the presence of $5 \times 10^{-4} \mathrm{M}$ unlabeled PCP. The total volume of the reaction mixture was $300 \mu \mathrm{l}$. Samples $(10 \mu \mathrm{l})$ were then filtered $(0,-U . V ., N o$ "dilution") or diluted 500 -fold and filtered after $60 \min (O,-U . V$., "dilution"). The rest of the mixture was irradiated under the standard conditions as described under "Materials and Methods." After photolysis, 10$\mu$ l samples were diluted 500-fold in Tris buffer and filtered 60 min later $(\triangle,+U . V$. . "dilution"). The rest of the membranes were centrifuged, and the pellets were resuspended and washed

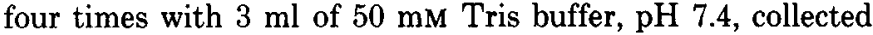
and counted $(\boldsymbol{\Lambda},+U . V$. ., "Washed" pellets $)$. Each data point represents the specifically bound ligand (i.e., total minus nonspecific binding).

the absence and in the presence of carbamylcholine and that the labeling of the 50,000-dalton subunit is enhanced by this cholinergic agonist.

\section{Discussion}

The cholinergic effects of PCP and its derivatives have been extensively described (Maayani et al., 1974; Paster et al., 1974; Kloog et al., 1977; Kalir et al., 1978; Petersen and Stillman, 1978). However, it was recognized only recently that these drugs may interact directly with the cholinergic ionophore (Kloog et al., 1979, 1980; Albuquerque et al., 1980; Eldefrawi et al., 1980; Oswald and Changeux, 1981a). Electrophysiological (Albuquerque et al., 1980) as well as binding studies (Eldefrawi et al., 1980; Kloog et al., 1980; Oswald and Changeux, 1981a) suggest that PCP acts as a noncompetitive blocker of AcCho. Thus, the drug appears to fall into the pharmacological category of local anesthetics (Webber and Changeux, 1974; Karlin, 1980) and related drugs (Elliott and Raftery, 1979; Albuquerque et al., 1980) rather than that of the cholinergic agonists and their competitive

rarine $+1 \mu \mathrm{M} \alpha$-Bgt ( $\square$ ). When $\alpha$-Bgt was used, the membranes were pre-incubated with the toxin for $30 \mathrm{~min}$ at room temperature. Decamethonium, hexamethonium, and $d$-tubocurarine were added 5 min prior to the addition of $\left[{ }^{3} \mathrm{H}\right] \mathrm{PCP}$. 


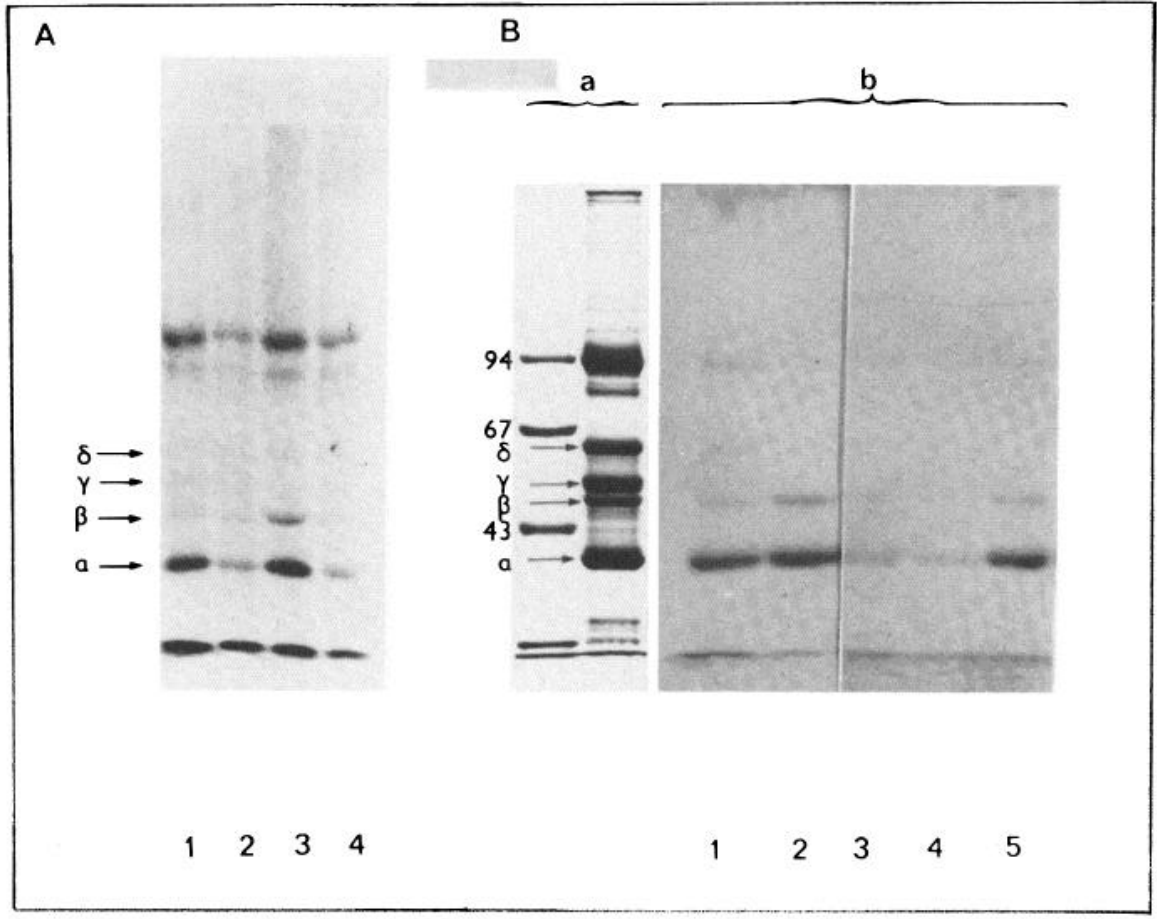

Figure 5. Fluorograms of SDS polyacrylamide gels of $\left[{ }^{3} \mathrm{H}\right] \mathrm{AZ}-\mathrm{PCP}$ photolabeled receptor-enriched membrane proteins. Membranes were incubated with $1.0 \mu \mathrm{M}$ $\left[{ }^{3} \mathrm{H}\right] \mathrm{AZ}-\mathrm{PCP}$ in the presence and absence of various agents, then irradiated and electrophoresed as described under "Materials and Methods." Each lane was loaded with $100 \mu \mathrm{g}$ of protein. The dry stained gels were then exposed to the $\mathrm{x}$ ray film for 30 days as described under "Materials and Methods." $A$, Membranes were incubated with $\left[{ }^{3} \mathrm{H}\right] \mathrm{AZ}-\mathrm{PCP}$ in the absence $(1)$ and in the presence of $10^{-3}$ M PCP (2), $10 \mu \mathrm{M}$ carbamylcholine (3), and $10 \mu \mathrm{M}$ carbamylcholine $+10^{-3} \mathrm{M}$ PCP (4). B, Coomassie blue staining pattern $(a)$ of photoinactivated membranes and of molecular weight standards electrophoresed in lanes parallel to those shown in the fluorogram $(b)$. Membranes were incubated with $\left[{ }^{3} \mathrm{H}\right] \mathrm{AZ}-\mathrm{PCP}$ in the absence (1) and in the presence of $100 \mu \mathrm{M}$ carbamylcholine (2), $10^{-3} \mathrm{M}$ tetracaine (3), $10^{-3} \mathrm{M}$ tetracaine $+100 \mu \mathrm{M}$ carbamylcholine (4), and $1.0 \mu \mathrm{M} d$ tubocurarine (5).

antagonists. The binding sites for the latter are located on the two 40,000-dalton $\alpha$ subunits of the nicotinic acetylcholine receptor (Karlin, 1980; Conti-Tronconi and Raftery, 1982).

To localize the sites on the AcChoR onto which PCP would bind, we synthesized an azido derivative of PCP and used it as a photoaffinity probe. Azido derivatives of drugs have the advantage that they can be examined under two sets of conditions: those in which they bind reversibly and those in which they irreversibly inactivate their binding sites. Their disadvantage is that incorporation of the azido group may interfere with the drug's specificity. Therefore, when using an azido derivative, it is important to start by examining its specificity (Bayley and Knowles, 1977; Zisapel and Sokolovsky, 1977).

In our detailed analysis of the binding of AZ-PCP, we demonstrated that its binding properties are almost indistinguishable from those of the parent molecule, PCP. That is, both drugs bind with high affinity to AcChoRenriched membranes from Torpedo electric organ, the number of specific binding sites is identical in both, their binding is inhibited by noncompetitive blockers of
AcCho, they competitively inhibit each other's binding, and their binding is similarly enhanced by cholinergic drugs like carbamylcholine and $d$-tubocurarine. These similarities confirm the suitability of AZ-PCP as a photoaffinity probe for PCP binding sites in Torpedo electric organ membranes.

In the absence of carbamylcholine, several polypeptides in the AcChoR-enriched membranes are labeled by AZ-PCP. Among them are the 65,000-, 55,000-, 50,000-, and 40,000-dalton polypeptides, which correspond, respectively, to the receptor subunits $\delta, \gamma, \beta$, and $\alpha$ (Karlin, 1980; Conti-Tronconi and Raftery, 1982). Two other labeled bands correspond to polypeptides with apparent molecular weights of 92,000 and 81,000 . The amount of labeling of these two polypeptides varied between experiments and was not affected significantly by cholinergic drugs or local anesthetics. The nature of their labeling is still unknown. The presence of these two polypeptides in receptor-enriched membranes from Torpedo electric organs, however, has been previously established (Karlin, 1980; Conti-Tronconi and Raftery, 1982). Unlike the labeling of these two polypeptides, labeling of the four 

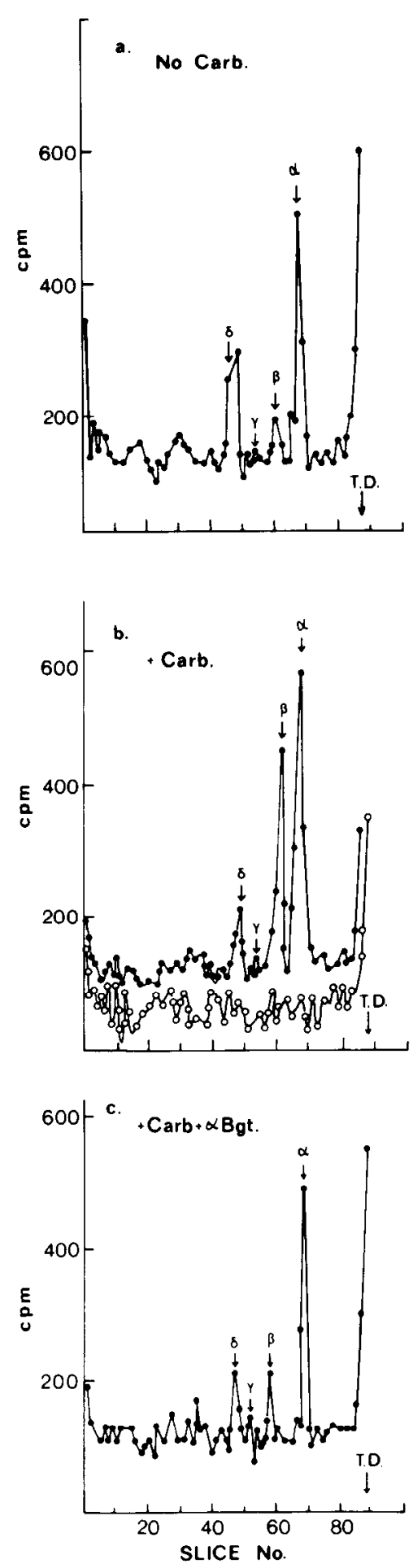

Figure 6. SDS polyacrylamide gel electrophoresis of $\left[{ }^{3} \mathrm{H}\right] \mathrm{AZ}$ PCP photolabeled receptor-enriched membranes. Membranes were treated as described for Figure 5, except that the gels were sliced and counted as described under "Materials and Methods." Incubations were under the following conditions: $a$, No carbamylcholine; $b,+100 \mu \mathrm{M}$ carbamylcholine (\$) or $+100 \mu \mathrm{M}$ carbamylcholine $+10^{-3} \mathrm{M}$ tetracaine $(\mathrm{O}) ; c,+100 \mu \mathrm{M}$ carbamylcholine $+10 \mu \mathrm{M} \alpha$-Bgt.

polypeptides which correspond to the AcChoR was consistently observed in all of our experiments.

By far the most heavily labeled band was that corresponding to the $\alpha$ subunit, which is known to carry the
AcCho binding sites (Karlin, 1980; Conti-Tronconi and Raftery, 1982). Labeling of this subunit was strongly inhibited by both PCP and tetracaine. The snake venom $\alpha$-Bgt, however, did not block labeling of the $\alpha$ subunit when used at a concentration $(1 \mu \mathrm{M})$ at which it completely blocks AcCho binding sites (Eldefrawi et al., 1982). Therefore, the binding of AZ-PCP to the $\alpha$ subunit must occur on sites other than the AcCho binding sites. Indeed, addition of carbamylcholine $(10 \mu \mathrm{M})$ did not affect labeling of the $\alpha$ subunit; however, it caused a strongly enhanced labeling of the 50,000-dalton polypeptide (the $\beta$ subunit). Labeling of the $\beta$ subunit was inhibited by PCP and tetracaine, and the effect of carbamylcholine was blocked by $\alpha$-Bgt. Therefore, we conclude that the $\beta$ subunit carries specific binding sites for PCP. Moreover, it is evident that the occupation of AcCho binding sites by carbamylcholine leads to enhanced binding of AZ-PCP to the $\beta$ subunit, probably via the known conformational changes induced by the agonist (Karlin, 1980; Conti-Tronconi and Raftery, 1982). Therefore, the observed increase in affinity of PCP to its binding sites is at least partly due to the stronger attachment of the ligand to the $\beta$ subunit in the presence of carbamylcholine than in its absence. In the absence of cholinergic agonist, the membrane-bound receptor is present mostly in its resting state, while in its presence it is in its desensitized form (Popot et al., 1976; Sugiyama et al., 1976). Thus, AZ-PCP binds preferentially to the $\alpha$ subunit when the receptor is in its resting state and to both the $\alpha$ and $\beta$ subunits when the receptor is in the desensitized state.

We consistently observed an enhanced labeling of the $\beta$ subunit in the presence of carbamylcholine. In earlier experiments, Oswald et al. (1980) demonstrated that labeling of a 50,000-dalton polypeptide by azido trimethisoquin was enhanced by carbamylcholine. They later showed that this labeled band was a proteolytic product of the $\delta$ subunit (Saitoh et al., 1980). In other studies, Oswald and Changeux (1981b) demonstrated that labeling of the $\delta$ subunit also occurs in the presence of other noncompetitive blockers of AcCho, such as perhydrohistionicotoxin $\left(\mathrm{H}_{12}-\mathrm{HTX}\right)$ and PCP. Although we did observe labeling of the $\delta$ subunit with AZ-PCP, we did not observe an increase in its labeling in the presence of carbamylcholine. This discrepancy cannot be explained by assuming the presence of proteolytic enzymes. We investigated the effect of using inhibitors of the four major classes of proteolytic enzymes, during both the membrane purification and the reactions. The latter were performed with and without enzyme inhibitors, and the results were the same in each case, both in binding experiments and on the gels. Purification of membranes in the absence of protease inhibitors resulted in the appearance of additional Coomassie blue-stained bands labeled by AZ-PCP at molecular weights lower than 40,000 (not shown). However, even under these conditions, labeling of the $\beta$ subunit was enhanced in the presence of carbamylcholine. If a highly active protease were present in our preparations, we should have seen at least a residual enhancement effect on the $\delta$ subunit when protease inhibitors were used, if this were the original target for AZ-PCP. Therefore, we suggest that the discrepancy between our work and that of Changeux 
may stem from species differences ( $T$. ocellata was used here, whereas the other study used Torpedo marmorata (Oswald and Changeux, 1981a, b).) Recent studies have indeed indicated that species differences determine the AZ-PCP labeling pattern in desensitized nicotinic acetylcholine receptors (Haring et al., 1983). Carbamylcholine enhanced the labeling of the $\beta$ subunit in T. ocellata, the $\delta$ subunit in T. marmorata, and the $\alpha$ subunit in Torpedo californica. Furthermore, Kaldany and Karlin (1983) have recently demonstrated that carbamylcholine enhances the labeling of $\alpha$ and $\beta$ subunits in T. californica by quinacrine mustard.

At the micromolar concentration range, binding of $\left[{ }^{3} \mathrm{H}\right] \mathrm{PCP}$ and $\left[{ }^{3} \mathrm{H}\right] \mathrm{AZ}-\mathrm{PCP}$ is saturable both in the presence and in the absence of carbamylcholine. We found no evidence supporting the existence of two or more binding sites for either labeled ligand. The labeling experiments clearly demonstrate, however, that at least the $\alpha$ and $\beta$ receptor subunits were labeled. One possible explanation for this apparent discrepancy is that the binding sites on the $\alpha$ and $\beta$ subunits are independent of each other, and in the absence of carbamylcholine the affinity of PCP toward both is similar and thus undetectable in our binding measurements. If PCP binds independently to $\alpha$ and $\beta$ subunits, an increase in maximal binding capacity should have been observed in the presence of carbamylcholine. That is, we should have seen binding to two populations of sites with low and high affinity, reflecting binding to $\alpha$ and $\beta$ subunits, respectively. We did not detect either an increase in the binding capacity or heterogeneity of binding. Therefore, it is unlikely that PCP at the micromolar range binds independently to $\alpha$ and $\beta$ subunits.

Another plausible explanation is that the PCP binding site is located in the vicinity of the physical connection between the two subunits, and that consequently the PCP molecule is attached simultaneously both to $\alpha$ and $\beta$ subunits. In proposing such a hypothesis, we assume that one moiety of the PCP molecule is attached to the $\alpha$ and the other to the $\beta$ subunit. Addition of carbamylcholine in this case should increase only the attachment between the $\beta$ subunit and its PCP moieties; that is, we should observe no increase either in the number of PCP binding sites or in heterogeneity, but only an increase in affinity. This was indeed the experimental finding, but we should keep in mind that the azido moiety (through which the drug is covalently attached to the receptor subunits) is localed on the phenyl ring. If our hypothesis is correct, this would suggest that there are nonidentical overlapping binding sites for PCP on the AcChoR subunits. The receptor subunits (at least $\alpha$ and $\beta$ ) would then require specific recognition sites for both the phenyl moiety and the other (cationic nitrogen) moieties of PCP. The high degree of amino acid sequence homology between the receptor subunits (Conti-Tronconi et al., 1982a) and the asymmetric structure of the pentameric AcChoR (Karlin, 1980) are compatible with our hypothesis.

In previous reports Oswald and Changeux demonstrated that radiolabeled azido trimethisoquin (1981a), $\mathrm{H}_{12}$-HTX, PCP, and trimethisoquin (1981b) label all of the AcChoR subunits. Like our results with AZ-PCP, in the absence of cholinergic ligand the $\alpha$ subunit was always the most heavily labeled band. Labeling of the $\alpha$ subunit with trimethisoquin was inhibited by carbamylcholine and erabutoxin $b$ (Oswald and Changeux, 1981b), but labeling of the $\alpha$ subunit with PCP was not affected by these ligands. These results are in agreement with our own finding that at the micromolar concentration range the attachment of PCP to the $\alpha$ subunit is not via AcCho binding sites. However, in view of the heavy labeling of the $\alpha$ subunit by all noncompetitive blockers of AcCho tested so far, we suggest that the $\alpha$ subunit must be considered as a target for these drugs. Accordingly, local anesthetics can also bind to PCP sites on the $\alpha$ subunit. This suggestion is supported by binding data indicating that all of the binding of any of the ligands can be displaced by the others. That is, PCP binding can be displaced by $\mathrm{H}_{12}$ - $\mathrm{H}^{\prime} \mathrm{TX}$ and local anesthetics (Albuquerque et al., 1980; Kloog et al., 1980) (see Fig. 4), and $\mathrm{H}_{12^{-}}$ HTX binding or meproadifen binding can be displaced by local anesthetics or PCP (Elliott and Raftery, 1979; Krodel et al., 1979; Albuquerque et al., 1980). Moreover, the number of the high affinity sites for meproadifen (Krodel et al., 1979), PCP (Kloog et al., 1980), and $\mathrm{H}_{12^{-}}$ HTX (Albuquerque et al., 1980) in receptor-enriched membranes is similar (the ratio of noncompetitive drug sites to $\alpha$-Bgt sites is between 0.25 and 0.35 ).

We have shown that cholinergic ligands, such as $\alpha$ Bgt and carbamylcholine, in concentrations at which they completely block AcCho binding sites, do not interfere with the high affinity PCP or AZ-PCP binding. However, we know that other cholinergic drugs, such as $d$-tubocurarine, at concentrations well above those needed to block AcCho sites, do block the high affinity PCP (Fig. 3) or $\mathrm{H}_{12}$-HTX binding (Shaker et al., 1982). Eldefrawi et al. (1982) also demonstrated that nicotine and decamethonium can interact with $\mathrm{H}_{12}$-HTX binding sites on the AcChoR when the AcCho sites are blocked by $\alpha$-Bgt. Therefore, it seems that several cholinergic ligands can also bind to PCP binding sites but with a lower affinity than to the AcCho sites. It should be noted that from other experiments we know that the $\alpha$ subunit can bind local anesthetics at the AcCho site. Meproadifen (Krodel et al., 1979), trimethisoquin (Oswald and Changeux, 1981a, b), and quinacrine (Tsai et al., 1979) are the best examples. It is interesting to note that Conti-Tronconi et al. (1982b) reported recently on a low affinity binding site for AcCho that is distinct from the high affinity AcCho binding site. The localization of the low affinity site is still unknown. Data presented here and by others indicating low affinity binding of cholinergic drugs to PCP or $\mathrm{H}_{12}$-HTX binding sites (Eldefrawi et al., 1982) open the possibility that these sites are the low affinity AcCho binding sites.

To summarize, the binding characteristics of noncompetitive blockers of AcCho as well as our photoaffinity labeling experiments suggest that at least two binding sites on the AcChoR are recognized by both the noncompetitive blockers and the cholinergic drugs. One of these is the well known AcCho site located on the $\alpha$ subunit. This site preferentially binds cholinergic drugs such as AcCho, $d$-tubocurarine, carbamylcholine, and $\alpha$-Bgt (Karlin, 1980; Conti-Tronconi and Raftery, 1982). It can also bind local anesthetics, such as meproadifen (Krodel et al., 1979), trimethisoquin (Oswald and Changeux, 
1981a), and possibly PCP as well, but with lower affinity. The second site on the AcChoR preferentially binds PCP and probably local anesthetics as well. It can also bind cholinergic drugs, but with lower affinity. According to our hypothesis, the second site is localized between the $\alpha$ and $\beta$ subunits of the AcChoR from T. ocellata. This model is similar to that described for the binding of phosphoglycerate to the two $\beta$ subunits of hemoglobin (Baldwin and Chothia, 1979). Since local anesthetics and related drugs exhibit many common features when acting on the AcChoR, we believe that such a model explains the interaction of all of them. However, differences between the actions of various local anesthetics and other channel blockers have also been reported (Eldefrawi et al., 1982). Thus, additional quantitative data are needed on the labeling of receptor subunits with PCP and local anesthetics.

It would now be of great interest to localize the polypeptides to which PCP binds in mammalian brain membranes. These membranes have been shown to possess high affinity binding sites for PCP (Vincent et al., 1979; Zukin and Zukin, 1979). If AZ-PCP binds like PCP to the high affinity PCP binding sites in brain membranes, it will be able to serve as a potent photoaffinity probe for these sites.

\section{References}

Albuquerque, E. X., M. C. Tsai, R. S. Aronstam, A. T. Eldefrawi, and M. E. Eldefrawi (1980) Sites of action of phencyclidine II. Mol. Pharmacol. 18: 167-178.

Amitai, G., S. Avissar, D. Balderman, and M. Sokolovsky (1982) Affinity labeling of muscarinic receptors in rat cerebral cortex with a photolabile antagonist. Proc. Natl. Acad. Sci. U. S. A. $79: 243-247$.

Aronstam, R. S., M. E. Eldefrawi, A. T. Eldefrawi, E. X. Albuquerque, K. F. Jim, and D. J. Triggle (1980) Site of action of phencyclidine III. Mol. Pharmacol. 18: 179-184.

Baldwin, J., and C. Chothia (1979) Haemoglobin: The structural changes related to ligand binding and its allosteric mechanism. J. Mol. Biol. 129: 175-220.

Bayley, H., and J. R. Knowles (1977) Photoaffinity labelling. Methods Enzymol. 66: 69-114.

Bonner, W. M., and R. A. Laskey (1974) A film detection method for tritium-labeled proteins and nucleic acids in polyacrylamide gels. Eur. J. Biochem. 46: 83-88.

Conti-Tronconi, B. M., and M. A. Raftery (1982) The nicotinic cholinergic receptor: Correlation of molecular structure with functional properties. Annu. Rev. Biochem. 51: 491-530.

Conti-Tronconi, B. M., M. W. Hunkapiller, J. M. Lindstrom, and M. A. Raftery (1982a) Amino acid sequence homology between " $\alpha$ " subunits from Torpedo and Electrophorus acetylcholine receptor. Biochem. Biophys. Res. Commun. 106: 312-318.

Conti-Tronconi, B. M., S. M. J. Dunn, and M. A. Raftery (1982b) Independent sites of low and high affinity for agonists on Torpedo californica acetylcholine receptor. Biochem. Biophys. Res. Commun. 107: 123-129.

Eldefrawi, M. E., A. T. Eldefrawi, R. S. Aronstam, S. E. Warnick, and E. X. Albuquerque (1980) $\left[{ }^{3} \mathrm{H}\right]$ Phencyclidine: A probe for the ionic channel of the nicotinic receptor. Proc. Natl. Acad. Sci. U. S. A. 77: 7458-7462.

Eldefrawi, A. T., E. Roxanne Miller, and M. E. Eldefrawi (1932) Binding of depolarizing drugs to the ionic channel sites of the nicotinic acetylcholine receptor. Biochem. Pharmacol. 31: 1819-1822.
Elliott, J., and M. A. Raftery (1979) Binding of perhydrohistionicotoxin to intact and detergent-solubilized membranes enriched in nicotinic acetylcholine receptor. Biochemistry 18: 1868-1874.

Gabrielevitz, A., Y. Kloog, A. Kalir, D. Balderman, and M. Sokolovsky (1980) Interaction of phencyclidine and its new adamantyl derivatives with muscarinic receptors. Life Sci. 26: 89-95.

Haring, R., Y. Kloog, and M. Sokolovsky (1983) Species differences determine azido phencyclidine labeling pattern in desensitized nicotinic acetylcholine receptors. Biochem. Biophys. Res. Commun. 113: 723-729.

Johnston, M., V. Evans, and S. Baigel (1959) Sernyl in clinical anesthesia. Br. J. Anaesth. 31: 433-439.

Kaldany, R. R. J., and A. Karlin (1983) Reaction of quinacrine mustard with the acetylcholine receptor from Torpedo californica: Functional consequences and sites of labeling. J. Biol. Chem. 258: 6232-6242.

Kalir, A., S. Sadeh, J. Karoly, E. Shirin, D. Balderman, J. Eolery, and G. Porath (1975) 1-Phenylcycloalkylamine derivatives. Part III. Isr. J. Chem. 13: 125-136.

Kalir, A., S. Maayani, M. Rehavi, R. Elkavetz, I. Pri-Bar, O. Buchman, and M. Sokolovsky (1978) Structure-activity relationship of some phencyclidine derivatives: In vivo studies in mice. Eur. J. Med. Chem. 13: 17-24.

Karlin, A. (1980) Molecular properties of nicotinic acetylcholine receptors. In The Cell Surface and Neuronal Function, C. W. Cotman, G. Poste, and G. L. Nicholson, eds., Vol. 6, pp. 191-260, Elsevier/North Holland Biomedical Press, New York.

Kloog, Y., M. Rehavi, S. Maayani, and M. Sokolovsky (1977) Anticholinesterase and antiacetylcholine activity of 1-phenylcyclohexylamine derivatives. Eur. J. Pharmacol. 45: 221227.

Kloog, Y., A Gabrielevitz, A. Kalir, D. Balderman, and M. Sokolovsky (1979) Functional evidence for a second binding site of nicotinic antagonists using phencyclidine derivatives. Biochem. Pharmacol. 28: 1447-1450.

Kloog, Y., A. Kalir, O. Buchman, and M. Sokolovsky (1980) Specific binding of $\left[\mathrm{H}^{3}\right]$ phencyclidine to membrane preparation. FEBS Lett. 109: 125-128.

Krodel, E. K., R. A. Beckman, and J. B. Cohen (1979) Identification of a local anesthetic binding site in nicotinic post synaptic membranes isolated from Torpedo marmorata electric tissue. Mol. Pharmacol. 15: 294-312.

Laemmli, U. K. (1970) Cleavage of structural proteins during the assembly of the head of bacteriophage $T_{4}$. Nature (Lond.) 227: 680-685.

Maayani, S. and H. Weinstein (1980) "Specific binding" of $\left[\mathrm{H}^{3}\right]$ phencyclidine: Artifacts of the rapid filtration method. Life Sci. 26: 2011-2022.

Maayani, S., A. Weinstein, N. Ben-Zvi, S. Cohen, and M. Sokolovsky (1974) Psychotomimetics as anticholinergic agents. I. 1-Cyclohexylpiperidine derivatives: Anticholinesterase activity and antagonistic activity to acetylcholine. Biochem. Pharmacol. 23: 1263-1281.

Ncubig, R. R., E. K. Krodel, N. D. Boyd, and J. B. Cohen (1979) Acetylcholine and local anesthetic binding to Torpedo nicotinic postsynaptic membranes after removal of nonreceptor peptides. Proc. Natl. Acad. Sci. U. S. A. 76: 690-694.

Oswald, R., A. Sobel, G. Waksman, B. Raques, and J. P. Changeux (1980) Selective labeling by $\left[\mathrm{H}^{3}\right]$ trimethisoquine azide of polypeptide chains present in acetylcholine receptorrich membranes from Torpedo marmorata. FEBS Lett. 111: 29-34.

Oswald, R. E., and J. P. Changeux (1981a) Selective labeling of the $\delta$ subunit of the acetylcholine receptor by a covalent local anesthetic. Biochemistry 20: 7166-7174. 
Oswald, R., and J. P. Changeux (1981b) Ultraviolet lightinduced labeling by noncompetitive blockers of the acetylcholine receptor from Torpedo marmorata. Proc. Natl. Acad. Sci. U. S. A. 78: 3925-3929.

Paster, Z., S. Maayani, H. Weinstein, and M. Sokolovsky (1974) Cholinergic action of phencyclidine derivatives. Eur. J. Pharmacol. 25: 270-274.

Petersen, R. C., and R. C. Stillman (1978) Phencyclidine: An overview. In Phencyclidine (PCP) Abuse: An Appraisal, National Institute of Drug Abuse Research Monograph 21, R. C. Petersen and R. C. Stillman, eds., pp. 1-27, National Institute of Drug Abuse, Rockville, MD.

Popot, J. L., H. Sugiyama, and J. P. Changeux (1976) Studies on the electrogenic action of acetylcholine with Torpedo marmorata electric organ. II. The permeability response of the receptor rich membrane fragments to cholinergic agonists in vitro. J. Mol. Biol. 106: 469-483.

Saitoh, T., R. Oswald, L. P. Wennogle, and J. P. Changeux (1980) Conditions for the selective labeling of the 66,000 dalton chain of the acetylcholine receptor by the covalent non-competitive blocker 5 -azido- $\left[\mathrm{H}^{3}\right]$-trimethisoquin. FEBS Lett. 116: 30-36.

Schmidt, J., and M. A. Raftery (1973) A simple assay for the study of solubilized acetylcholine receptors. Anal. Biochem. 52: 349-354.

Shaker, N., A. T. Eldfrawi, L. G. Aguayo, J. E. Warnick, E. X. Albuquerque, and M. E. Eldefrawi (1982) Interactions of $d$ tubocurarine with the nicotinic acetylcholine receptor/chan- nel molecule. J. Pharmacol. Exp. Ther. 220: 172-177.

Sugiyama, H., J. L. Popot, and J. P. Changeux (1976) Studies on the electrogenic action of acetylcholine with Torpedo marmorata electric organ. III. Pharmacological desensitization in vitro of the receptor-rich membrane fragments by cholinergic agonists. J. Mol. Biol. 106: 485-496.

'I'sai, M. C., A. C. Oliviera, E. X. Albuquerque, M. E. Eldefrawi, and A. T. Eldefrawi (1979) Mode of action of quinacrine on the acetylcholine receptor ionic channel complex. Mol. Pharmacol. 16: 382-392.

Vincent, J. P., D. Carrey, J. M. Kamenka, P. Geneste, and M. Lazdunski (1978) Interaction of phencyclidine with the muscarinic and opiate receptors in the central nervous system. Brain Res. 152: 176-182.

Vincent, J. P., N. Kartolovsky, P. Geneste, J. M. Kamenka, and M. Lazdunski (1979) Interaction of phencyclidine ("angel dust") with a specific receptor in rat brain membranes. Proc. Natl. Acad. Sci. U. S. A. 76: 4678-4682.

Webher, M., and J. P. Changeux (1974) Binding of Naja nigrocollis $\left[\mathrm{H}^{3}\right] \alpha$-neurotoxin to membrane fragments from Electrophorus and Torpedo electric organs. III. Effects of local anaesthetic on the binding of the tritiated $\alpha$-neurotoxin. Mol. Pharmacol. 10: 35-40.

Zisapel, N., and M. Sokolovsky (1977) Affinity labeling of receptors. Methods Enzymol. 66: 572-581.

Zukin, S. R., and R. S. Zukin (1979) Specific $\left[\mathrm{H}^{3}\right]$ phencyclidine binding in rat central nervous system. Proc. Natl. Acad. Sci. U. S. A. $76: 5372-5376$. 